\title{
External Fixation of Intertrochanteric Fractures In High Risk Geriatric Patients Done Under Local Anaesthesia
}

\author{
Dr Naveed Bashir wani, Dr A R Badoo. \\ ${ }^{I}$ Registrar, Department of Orthopaedics, Hospital for Bone \& Joint Surgery, Barzulla, Srinagar, associated \\ hospital GMC Srinagar J\&K. \\ ${ }^{2}$ Associate Professor and Unit head, Department of orthopaedics, Hospital for Bone and Joint Surgery, \\ Barzullah Srinagar, associated hospital GMC Sringar.
}

\begin{abstract}
:
Objective: The purpose of this study is to evaluate the results of external fixation of intertrochanteric fractures in very highrisk geriatric patients done under local anaesthesia.

Design: Prospective clinical study.

Settings: Level-1 trauma Centre.

Patients: forty eightvery high anaesthetic/surgicalrisk geriatric patients with intertrochanteric fractures. Intervention: Close reduction and external fixation using local anaesthesia supplemented by analgesics. Outcome Measured: Fracture Union, Time To Union, Pin Tract Infection Rate And Return To Daily Activities. Results: Forty eight patients with average age of 79.5 years all high anaesthetic/surgical risk were managed by closed reduction and external fixation under local anaesthesia supplemented by analgesics. Sixteen patients died before fracture union, seven were lost in follow up after removal of fixator and in one patient fixator was removed early after loss of reduction. Remaining twenty four were in regular follow up for at least six months. Pin tract inflammation was the most common complication while shortening andvarus collapse occurred in twelvecases.
\end{abstract}

Conclusion: external fixation of intertrochanteric fractures is useful in high risk geriatric patients and can be done under local anaesthesia supplemented by analgesics.

Keywords: intertrochanteric fractures, external fixation, local anaesthesia.

\section{Introduction}

Hip fractures are a leading cause of death and disability among the elderly ${ }^{1}$.These fractures include femoral neck and intertrochanteric fractures. Intertrochanteric fractures generally occur as a result of low energy trauma in advanced age, whereas they are caused by high energy trauma in young age ${ }^{2}$. The incidence of intertrochanteric fractures is increasing day by day as is the life expectancy. Life time risk in industrialized countries of intertrochanteric fractures is $6 \%$ for men and $18 \%$ for women. ${ }^{3}$ Thus we have old patients, many of them with co morbidities, having intertrochanteric fractures. Treatment goals in this population include early rehabilitation, restoration of the anatomic alignment of the proximal part of the femur and maintenance of the fracture reduction. ${ }^{4}$

Various treatment methods used for intertrochanteric fractures include operative and non-operative ${ }^{5}$. Historically, non-operative management took one of the two different protocols. In first approach, directed at early mobilization within the limits of the patient discomfort, the patient is allowed out of bed and in chair within a few days of injury. Ambulation was delayed, but the early bed to chair mobilization helped prevent many complications of prolonged recumbence. This approach did not attempt to treat the fracture specifically and accepted the deformity that invariably ensues. The second approach, in contrast, attempted to establish and maintain a reasonable reduction via skeletal traction (Hamilton Russel traction) until fracture union occurred. This technique was prolonged with difficulty in maintaining the reduction; further all the complications of prolonged recumbence like pressure sores, urinary tract infection, deep vein thrombosis, pulmonary embolism, chest infection are there. Operative methods include open reduction and internal fixation by sliding hip screw and its variants, intramedullary devices like gamma nails, PFN and their variants. Operative methods are treatment of choice in such fractures with their own advantages and disadvantages for particular implants. The sliding hip screw is widely used for fixation of peritrochanteric fractures and it may be considered as gold standard for both stable and unstable fractures. ${ }^{6}$ However, in many situations it is not feasible to go for open reduction and internal fixation in patients because of poor health and very high surgical/anaesthetic risks. In such patients conservative method by traction (Hamilton Russel traction) is an option. It has very high rates of 
complications because of prolonged recumbence ${ }^{7}$. In such situations closed reduction and external fixation is an option which may be considered as semi conservative method of treatment. Anderson et al used external fixators for the treatment of intertrochanteric fractures for the first time in $1943^{8}$ The advantages of external fixators in treatment of intertrochanteric fractures, have since been recognised by several authors ${ }_{1,3,7,9,16,18,20}$. Authors have been using external fixators for managing intertrochanteric fractures for many years with good results ${ }^{10}$. However, most of these cases were done under spinal or epidural anaesthesia. Only a few of these cases, who were very high risk even for spinal/epidural anaesthesia, were done under local anaesthesia. Encouraged by their results we started doing more and more of such high risk patients under local anaesthesia supplemented by analgesics. We used AO external fixators under local anaesthesia in the treatment of intertrochanteric fractures in elderly patients who were otherwise very high surgical/ anaesthetic risks and evaluated the results to assess this treatment option in this patient group.

\section{Material And Methods}

We evaluated the results of 48patients treated by AO unilateral external fixator for intertrochanteric fractures between September 2012 and September 2013 at our hospital. All patients with intertrochanteric fractures who were of > 65years age, having other systemic illnesses, poor health, which would make them very high surgical/ anaesthetic risk were included. Patients with dementia, poly trauma and patients who were not independent ambulators prior to fall or who had pathological fractures were excluded. The average age was 79.5 years (range 65 to 96 years).There were 28 males and 20 females. The average age of males was 83 and females 74.5 years.The inclusion of patients was based on anaesthetic/surgical risks rather than fracture classification. The study was approved by the hospitals scientific committee.

All patients were operated upon within 3 days of admission to the hospital. Thirty four (71\%)patients reached hospital on the day of trauma others on an average of 4 days, range (2 to 9) after trauma. Patients were operated on a fracture table under fluoroscopic control. All patients were operated under local anaesthesia supplemented by analgesics. Injection diclofenac was given as i/v bolus 20 minutes before surgery. Patients with impaired renal functions were given $\mathrm{i} / \mathrm{m}$ tramadol about half an hour before surgery. Patients were properly positioned and draped and closed reduction done. The quality of reduction was checked and accessed by as per criteria described by Baumgartner et al (for a reduction to be considered good, there had to be normal or slight valgus alignment on AP radiograph, less than 20 degrees angulations on lateral and no more than $4 \mathrm{~mm}$ displacement of any fragment, to be considered acceptable, a reduction had to meet the criterion of a good reduction with respect to either alignment or displacement, but not both. A poor reduction met neither criterion. ${ }^{11}$ Our aim was to achieve acceptable reduction within tolerable pain limits. Once reduction was achieved it was maintained by unilateral $\mathrm{AO}$ fixator using 4 pins. The proximal two pins were put in the region of greater trochanter through neck into head and were advanced to within 5-10 mm of joint surface. Distal two pins were put in shaft bicortically. Only stab wounds were used at previously infiltrated sites, pin sites were dressed with betadine dressing. Patients were encouraged to sit in a chair and stand using a walker on the day of surgery. They were taught pin site care and discharged next day with the instructions of pin site care, quadriceps exercise, and range of motion (ROM) exercise of knee, limited weight bearing and walking on frame. First visit was done after one week and then monthly or as required. On each follow up patients were evaluated for pin tract inflammation (PTI), ROM at knee, limb length discrepancy and radiologically for varus collapse and signs of union. PTI were graded according to Checkets and Otterburn ${ }^{12}$. Fixators were removed when signs of union appeared on X- rays (presence of trabeculae bridging the fracture site or obvious periosteal callous) usually $12-16$ weeks. Final follow up was at six months though there are patients with more than two years follow-up.
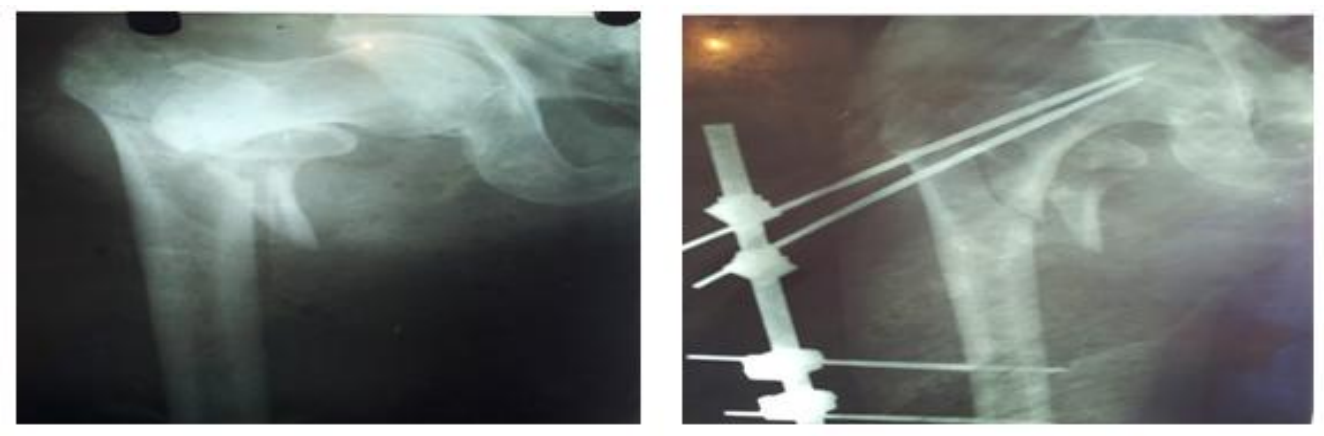

Figure 1a preoperative $\mathrm{X}$ ray showing intertrochanteric fracture $1 \mathrm{~b}$ immediate post-operative $\mathrm{X}$-ray showing external . fixator in place. 

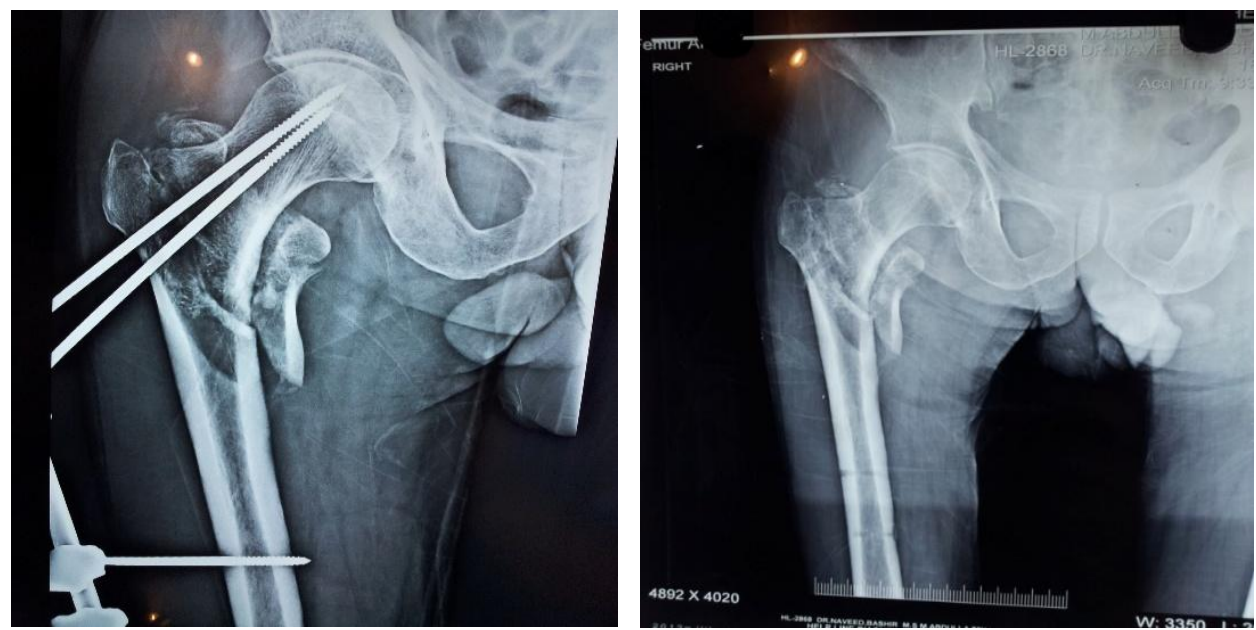

1c fixator in place during follow-up

$1 \mathrm{~d} X$ ray after removal of fixator at six months

\section{Results}

There were sixteen $(33 \%)$ deaths before removal of fixators. These deaths were due to medical ause unrelated to surgical intervention. Of the remaining thirty two patients thirty one united (97\%). Eight (17\%) patients were lost after removal of fixatorand never turned up again and as such their results cannot be commented upon. Fixators were removed at 12-16 weeks in $31(97 \%)$ patients and at6 weeks in one (3\%). In one patient fixator was removed at 6 weeks because of loss of fixation, he was offered repeat surgery after explaining risks which the family declined and was taken home with no further follow-ups. The time to union in our study was 12 to16 weeks (average 14.25 weeks).

Good reduction was achieved in 20 (41\%) patients and acceptable in 28 (59\%). No death or significant intra-operative complication occurred in any patient. No blood transfusion was needed intra or post operatively. Hospital stay for patients was two to four days with a mean of 2.5 days. PTI occurred in thirteen(42\%) patients. All of them settled with oral/iv antibiotics and local care. At six months twenty four patients were present for evaluation. Most of the patients had some amount of knee stiffness at the time of removal of fixator. At six months twelve (50\%) had knee flexion less than full and out of them six (25\%) had less than 90 degree of knee flexion. At six months post-operative $12(50 \%)$ out of 24 available for follow up had shortening $>2$ centimetres (cms) and radiologically varus $>20$ degree. $17(75 \%)$ were using some sort of support for walking, whereas $3(12.5 \%)$ were reluctant to walk even with support though their fracture had united.

\section{Complications}

No major intra operative complication/death occurred in our study. Pin tract infection (PTI) was the most common complication in our study occurring in $13(41 \%)$ of 31 patients, early mortality group and loss of fixation one excluded. All settled with oral/ iv antibiotics and local care. One patient with loss of reduction needed removal of fixator. Other common complications include stiffness of knee, varus collapse and limb shortening.

\section{Discussion}

The purpose of the study was to evaluatefeasibility of external fixation of intertrochanteric fractures in very high risk patients done under local anaesthesia. Thehip fracture is an injury and a disease at the same time. ${ }^{13}$ Charlsons co morbidity index, which predicts death in hospitalized elderly patients with fragility fractures at one year, for hip fractures is on an average 3.4 which corresponds to $52 \%$ of mortality at one year. ${ }^{14}$ Intertrochanteric fractures are one of the most important fractures and their incidence is increasing due to increase in life expectancy and osteoporosis. Their incidence is expected to double for each decade beyond the age of 50 years $^{15}$. These fractures usually occur in elderly patients who have other co- morbidities. All comorbidities benefit from early operation and mobilization. Keeping in view the life expectancy, the first goal is patient survival. $^{16}$

Treatment in intertrochanteric fractures ranges from conservative to operative. Achieving union is not a problem, since intertrochanteric fractures are through vascular cancellous bone. The problem is to maintain or 
reconstitute the neck shaft angle. The best a surgeon can achieve is to secure this position while allowing the patient to enjoy early ambulation. ${ }^{17}$ Operative treatment is the treatment of choice and DHS is the implant of choice for intertrochanteric fractures both stable and unstable,but there are situations where ORIF is not feasible owing to patient conditions. In these patients an option is conservative treatment (modified Russel traction) which has very high complication rates ${ }^{7}$. Keeping in view the rate of complications associated with conservative treatment and the high surgical risk associated with operative treatment a middle path is needed. A surgical procedure that has minimal surgical blood loss, short operating time and hospital stay, minimal anaesthetic risks, least morbidity and mortality rate and early weight bearing is favoured.$^{19}$ External fixators can be considered as a semi conservative method. ${ }^{20}$ External fixation gives a stable fixation, allows early mobilization thus preventing the complications of prolonged recumbence. External fixation is usually done under spinal/ epidural anaesthesia. But sometimes even spinal/epidural anaesthesia is not feasible and carry high risk. In such patients local anaesthesia has been tried with good results. The author has done a similar series were external fixation was done under spinal/epidural anaesthesia and few cases were done under local anaesthesia. Encouraged by those results we started doing more and more cases under local anaesthesia. The selection of patients for the procedure was based on poor state of general health producing very high risk.

In our study we had 33\% mortality within three months and further 17\% patients did not turn up for follow up after removal of frame and as such their survival remains doubtful. Higher mortality rate in our study may be due to very high risk patients. Other studies have mortality ranging from 14 to $39 \%{ }^{8,16,14}$ The union rate in our study was $97 \%$ excluding the early mortality group and the time to union was 14.25 weeks which is comparable to other studies. ${ }^{7,9,16,18}$ one patient had loss of reduction at six weeks. This patient had a reverse oblique fracture, which is a very unstable fracture, not feasible for external fixation. PTI was the most common complication though they subsided with antibiotics and local care. It occurred in out of 13 (42\%) of reported patients. Other's reported PTI ranging from $7 \%{ }^{16}$ to $32 \% .{ }^{18}$ It was superficial in most of the cases. Pin loosening due to infection was seen in 3 patients at around 6 to 8 weeks post-operative. They were managed by antibiotics, local care and limited weight bearing to prevent varus but all of them had $>20$ degree varus at union. The large variation in the number of PTI in different studies may be due to different criteria of recording PTI. Another cause of variation may be patient/attendants education level and his/her motivation to pin site care. All PTI in our study were controlled by local care and antibiotics.

Other complications include stiff knee, shortening, varus collapse. As far as knee stiffness is concerned it was present in most of the patients at the time of removal of frame but improved in most of them over a period of time. At six months only 6(25\%) had less than 90 degree knee flexion. Though knee flexion beyond 90 degree is not of much concern but in our part of world where people sit cross legged and squat some difficulty in daily living was present in them. Varus $>20$ degree along with shortening $>2 \mathrm{~cm}$ has been reported to occur in all major studies. Most studies report around 10 to $15 \% \cdot{ }^{16,18}$ Varus $>20$ degree and associated shortening $>2$ cms was present in twelve (50\%) of patients but was of little concern for the patients. Most of them had acceptable reduction with some varus to begin with, unstable fractures or PTI in postoperative period and loosening. Dhal ${ }^{7}$ explained it as due to either fracture fixation in varus or collapse of neck shaft angle on weight bearing. In our study in was due to both. Obtaining a perfect reduction of intertrochanteric fracture may not be the main objective for this high risk group but to operate with least blood loss in shortest time and an anaesthetic technique of low risk, which ultimately facilitates early mobilization in the post-operative period, may be preferred. When we consider the patient sub group in whom this method was used the results may be considered as good.

\section{References}

[1]. AntanioMoroni, Cesare Faldini, Francesco Pegreffietal Dynamic hip screw compared with external fixation for treatment of osteoporotic peritrochanteric fractures; jbjs volume 87-ANumber 4- April 2005.

[2]. Laskin L. S., Gruber M. A., ZimmmermanA.j. Intertrochanteric fractures of the hip in the elderly.

[3]. A retrospective analysis of 236 cases. Clin. Orthop., 1979, 141, 188-195.

[4]. Gillespie WJ. Hip fractures. BMJ 2001; 322; 968-75.

[5]. Baumgaertner MR. The peritrochanteric external fixation reduced pain, hospital stay, and mechanical complications in comparision with the sliding hip screw. Jbjs Am. 2002; 84; 1488.

[6]. Keneth J Koval, Robert V. Centu; intertrochanteric fractures. Rockwood and Green's fractures in Adults $6^{\text {th }}$ edition, chapter 45.

[7]. Hardy D.C.R., Descamps P.Y., Fabeck L., Simets P., Bertens C. L., Delince P. E. Use of an intramedullary hip screw compared with a compression hip screw with a plate for inter trochanteric femoral fractures. A prospective, randomised study of one hundred patients. J.B.J.S., 1998, 80-A, 618-30.

[8]. Anil Dhal, Mathew Vargese, V.B. Bhasin. External fixation of intertrochanteric fractures of femur. JBJS (Br) 1991; 73-B; 955-8

[9]. Anderson R., Mckibbin W B., Burgess E. Intertrochanteric fractures. JBJS, 1943, 25, 153-168. 
[10]. C. Vossinakis, L. S. Badras. The external fixator compared with the sliding hip screw for peritrochanteric fractures of femur. JBJS (Br) 2002; 84-B; 23-9.

[11]. Badoo A.R, Naveed Bashir, Syed BasitShafi Shah, M. Ahsan. External fixation of intertrochanteric fractures as an option in high risk geriatric patients. Jemds2015; Vol. 4, Issue 27, April 02; page: 4629-4635.

[12]. Baumgaertner MR, Curtin SL, Lindskog DM, Keggi JM. The value of the tip apex distance in predicting fauilure of fixation of peritrochanteric fractures of the hip. JBJS (Am), 1995; 77; 1058-64.

[13]. Checketts RG, Otterburn M. Pin tract infection; definition, prevention, incidence. In; abstracts of the $2^{\text {nd }}$ Riva congress, current perspectives' in external and intramedullary fixation. 1992 May 27-31; Riva di Garda, Italy. Sponsored by the university of Verona and the university of Montpellier; 1992. P 98-9.

[14]. Radkokomadina. The hip fracture is an injury and a disease at the same time. ZdravVestin; 77; iv -3-10.

[15]. Charlson M, Sazatrowski TP, Peterson j, Gold j. Validation of a combined comorbidity index. J ClinEpidemiol 1994; 47; $1245-51$.

[16]. Tak-Chuen, Yi Chiu, Wai-Leuk Tsang, Wai-leung, Sai-Hung Yeung. A double blinded, prospective, randomised, controlled clinical trail of minimally invasive dynamic hip screw fixation of intertrochanteric fractures. Injury,int. J. Care injured 40(2009) 422-427.

[17]. M. Subasi, C. Kesemenli, A. Kapukaya, S. Necmioglu. Treatment of intertrochanteric fractures by external fixation. ActaOrthopaedicaBelgica, vol. 67-5-2001.

[18]. Esser MP, Kassab JY, Jones DHA. Trochanteric fractures of femur. JBJS (Br) 1986; 68-B; 557-60.

[19]. George W. Boghdady. Mohammed Shalaby. Safety and reliability of external fixation for basicervical and intertrochanteric fractures in high-risk elderly patients. Strat trauma limb recon (2007) 2; 83-89.

[20]. Tomak Y, Kacaoglu M, Piskin A et al (2005). Treatment of intertrochanteric fracturesin geriatric patients with a modified external fixator. Injury 36; 635-43.

[21]. Eksioglu F., Gudemez E., Cavusoglo T., Sepici B. Treatment of intertrochanteric fractures by external fixation. Bull. Hosp. joint. Dis., 2000, 59, 131-135. 